\title{
Research on Multi-sensor Cooperative Tracking Mission Planning of Aerospace Hypersonic Vehicles
}

\author{
Qiang Fu, Chengli Fan*, Gang Wang and Xiangke Guo \\ School of Air And Missile Defense, Air force Engineering University, Xi'an 710051, China \\ ${ }^{*}$ Corresponding author
}

\begin{abstract}
Aimed at aerospace hypersonic vehicles (AHV) with the characteristics of high velocity, maneuverability, Radar Cross-section (RCS) weak, the single sensor is difficult to effectively track, therefore proposed multi-sensor collaborative workflow, construct cooperative tracking mission planning framework based on multi-agent system (MAS), and then multi-sensor cooperative optimization model is established. Proposed collaborative tracking mission planning algorithm based on Self-adaptive clonal genetic algorithm (SCGA). Simulation results validate the model, algorithm to establish is rationality and superiority.
\end{abstract}

Keywords- Aerospace hypersonic vehicles; multi-sensors; cooperative tracking; mission planning; self-adapting clonal genetic algorithms

\section{INTRODUCTION}

AHV will become a new strategic threat in the future, because of its high speed, super maneuverability, wide range of motion, working in the upper air and having a good stealth performance ${ }^{[1-2]}$.So, from a defense standpoint, it is the key and urgent technology to against the AHV that detecting fast, tracking steadily and continuity and identifying precisely toward the AHV ${ }^{[3-4]}$.

But for the limit of the sensor capability and the large span of time and space about the motion range of AHV, at present, it is difficult for a single sensor to complete the above-mentioned tasks independently. Therefore, it is an efficient measure to solve said problems that comprehensive utilization of all kinds of the observed information from all kinds of the sensors (such as infrared information from space-based sensor, visible light image information, target information from ground-based radar, and so on), as well as the detection and tacking based on the coordinated operation of heterogeneous sensors mission planning.

At present the research about multi-sensors collaborative tracking mission planning focused on the design of collaboration algorithm, multi-sensors management and resources scheduling ${ }^{[5-7]}$. In reference ${ }^{[6]}$, the presented scheme that the conjunction of sensor management strategy and motion dynamics guarantees a superior tacking performance. And it has made significant progress in the field of robotic kinematics. In reference ${ }^{[7]}$, it supposes that the conditions of the hardware configuration are superior. So it can be realized that multi-sensors track different targets by dynamic allocating the different sensor resources.
And it is helpful to the sensor management on cooperative tracking of AHV.

So, the insufficient factors about tracking the AHV by ground-based radar are analyst in this article. And the cooperative tracking capability of space-based multi-sensors platform is also be verified, which could provide supports for the ground-based sensor platform.

On this basis, the multi-sensors mission planning model of cooperative tracking is set up, which respects the characteristics of AHV. And then, the optimal operation of sensor resources based on SCGA (introducing the clonal selection and adaptive strategy in GA) is given. Simulation results show that this algorithm is effective and better than the ordinary one.

\section{ANALYSIS OF Multi-SENSORS COOPERATIVE TRACKING PlanNING BASED ON MAS}

Due to the characteristics of AHV,For all kinds of sensors of different platform, band, working mode, the detection performance of cooperative tracking task planning autonomy, interactivity, real-time, intelligence put forward higher requirement. As shown in figure 1, the distributed mission planning framework about multisensory synergistic tracking based on the MAS is designed.

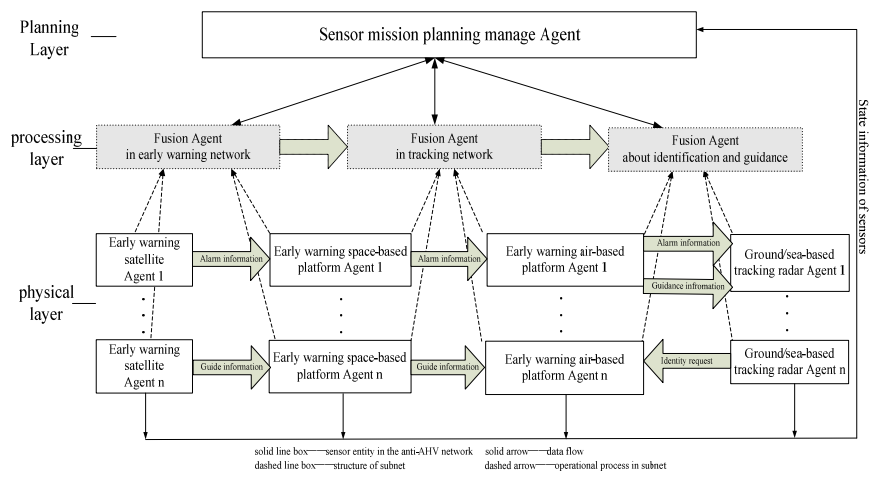

FIGURE I. FRAMEWORK OF MULTI-SENSOR COOPERATIVE TRACKING PLANNING BASED ON MAS

The characteristics of framework are hierarchical processing,distributed fusion and dynamic feedback fusion. The outstanding feature is to finish the dynamic resources scheduling of the single sensor and the construction and 
cooperative combat of the multi-sensors conveniently. And the specific characteristics as follow:

\section{(1) The intelligent synergetic operation}

Firstly, task planning and management are operated in the Planning Layer. And then the fusion subsystem is built up dynamically in Processing Layer. Thirdly, resources are scheduled in Physical Layer. According to the fusion result, guiding requirements and the feedback of the statement, the project of the task planning would be adjusted. Therefore, it needs all kinds of sensors work synergistically ${ }^{[10]}$. And the task planning synergetic question that need to be solved are multisensory Agent's transfer mode, transfer area, manner of working and time of duration, and so on.

(2) The structure of the agent of dynamic fusion processing system

Most of the agents of air-space sensors can structure subsystem agent of multitask, recognition, tracking and early warning. And according to the synergistic solution of task planning and the state parameter of different sensor, the suit sensors may be selected. As a result, the efficient network dynamically can be realized ${ }^{[11]}$. Base on the expandability and robustness of the framework of MAS, each sensor can build up the subsystem Agent effectively to carry out the task of recognition, tracking or early warning.

(3) Scheduling dynamically the resources of the sensors

When many sensors in the Physical Layer undertake the early warning task, they are also needed to carry out other tasks about recognition or tracking synergistically. Therefore, it is the key problem in the integration framework how to dynamically distribute the resources of sensors $^{[12-13]}$. Many-to-many task allocation mechanism is used in air-space high-speed flight vehicle synergetic tracking. It has its own specific cost function and task allocation matrix. So the benefit modes of Space-AirGround multi-sensors about corresponding recognizing, detect and tracking must be built. And the final judgment indexes would be given by utilizing the cost function and efficiency function.

\section{Mission Planning Model of SENSOR COLLABORATIVE TRACKING}

\section{A. Mathematical Description of the Problem}

It is assumed that the number of detection platforms about space-based high obit ${ }^{[14]}$, space-based low obit and ground-based platform is $m$. The platform number set is $\mathbf{I}=\{1,2,3, \cdots, m\}$. Each platform has $k_{i}, k_{i} \in\{1, \cdots, s\}$ sensor payloads. In the detecting and tracking area, the number of targets is $n$. And target label set is $\mathbf{J}=\{1,2,3, \cdots, n\}$. The priority evaluation of targets is $p_{j},(j \in \mathbf{J})$. The shortest time when sensor $i$ tracks the target $j$ is $T_{i j}$, and $t_{j o} \sim t_{j 1}$ is the time radian that target $\mathrm{j}$ from discovering to disappeared (depend on the ability of sensor and target track). If the sensor $i$ is tracking the target $j, C_{i j}=1$. Else $C_{i j}=0 . t_{i j 0} \sim t_{i j 1}\left(t_{j 0} \leq t_{i j 0}, t_{i j 1} \leq t_{j 1}\right)$ is the time radian during the target $j$ pass through the detecting range of sensor $i$. The whole tracking time from sensor discovers the first target to the last target runs out the detecting range of sensor is $t_{a}$. It's assumed that the completion time is $t_{g}$, and the sensor's breakdown and switchover time is ignored. When a new target appears, the new scheduling will be carried out. On the condition that important targets should be detected preferentially, trying to make sure that tracking each target.

\section{B. Objective Function}

The object of coordinated schedule about multi-sensor is that maximizing the tracking success rate $e$ and using rate $\mu$ of sensor, optimizing tracking performance $q$, as well as minimize sensor's switching rate $\eta^{[15]}$. And the objective function is given by weighting them, which is shown in formula (1). Normally, specific weighting can be given by analytic hierarchy process (AHP) or simulating optimization.

$$
f=w_{1} \cdot e+w_{2} \cdot q+w_{3} \cdot \mu-w_{4} \cdot \eta
$$

The factors such as penetration ability, hit accuracy, striking power, arrival time decide the threat level of AHV. The higher degree of threat, the higher priority of tracking. As a result, more resources are needed in order to maximize the whole probability of tracking. It is assumed that there are $k$ targets are tracking, and the success rate of tracking is as follows,

$$
e=\sum_{j=1}^{k} p_{j} / \sum_{j=1}^{n} p_{j}
$$

In the detecting and tracking of AHV, the tracking accuracy is the most important optimizing index, because of it influences the efficiency of synergetic detection and tracking. In a certain observation time, the choice of sensor combination has no effect on the estimation of current velocity, but for the estimation error of the position. So, according to the literature ${ }^{[16]}$, the estimation error of position in the covariance matrix can be the only one cared to optimize tracking accuracy of target. And the optimization method based on GDOP may have the same result that based on information entropy and covariance control. Therefore, GDOP is select as optimizing index of tracking accuracy. The identify of GDOP is as follows,

$$
G D O P=\sqrt{\operatorname{Tr}\left(\bar{P}_{k / k}\right)}
$$

The goal of minimize GDOP is to make the precision of the positioning highest. During tracking time, the accuracy factor of tracking $\mathrm{n}$ targets is defined as follow,

$$
q=\sum_{j=1}^{n} p_{j} G D O P(j)
$$


It is expected that each sensor can be maximum utilized during the total tracking time. The ratio between the time each sensor takes up and the total tracking time can be obtained by scheduling algorithm. And then, the use ratio of sensor can be given by averaging, as it is shown in the formula (5).

$$
\mu=\sum_{i=1}^{m} \sum_{k=1}^{s} t(i, k) / \sum_{i=1}^{m} \sum_{k=1}^{s} t_{a}
$$

Although the consuming time when switching sensors is not considered in the algorithm, the times of switching sensors may reflect the efficiency of tracking. It is best to reduce to times of switching sensor. The more number of $O(i, k)=0$ in the tracking list, the fewer times of switching sensors $\left(O_{m s}=1\right)$. The ratio of switching sensors is as follow,

$$
\eta=\sum_{i=1}^{m} \sum_{k=1}^{s} O(i, k) / \sum_{i=1}^{m} \sum_{k=1}^{s} O_{m s}
$$

\section{The Algorithm of Multi-Sensor CoOperative TRACKING MISSION PLANNING}

The model built above is a single nonlinear objective optimization problem under constrains. Although the basic genetic algorithm (GA) can find the satisfactory solution in a certain period of time, it doesn't make sure that the optimal solution of the problem can be given in real time ${ }^{[17-}$ 18]

Combined with the characteristics of antibody diversity an immunological memory in biological immune system, clone selection and adaptive strategy are introduced into GA. This algorithm called SCGA is used to solve the problem about optimal scheduling the sensor resources.

\section{A. Algorithm Design}

\section{1) Genetic encoding}

In order to maximize the sensor utilization, the genetic encoding is as the sensor. Chromosome is constituted by $m$ sub-chromosomes, and each sub- chromosomes is a $2 \times n$ dimensional array. Target observation number is represented by the first row of sub-chromosomes, and the corresponding observation time is represented by the second row. Through binding the target number with observation time, the onedimensional array is given. The odd number bits refer to target number, while even number bits refer to observation time. The length of chromosome is $2 \cdot m \cdot n$. The value range of observation sequence is from 0 to $n$, and the value of observation time is integer times per unit time. Both use integer coding. When the value of observation sequence number is 0 , it refers that the sensor would not tracking the target and the statement of the sensor is idle.

\section{2) Genetic operator}

1 Selection operator
According to the probability $p_{c}$, the individual in parent generation would be selected and then copied into the offspring one. The Baker linear selection probability is used as follow,

$$
p_{i}=\frac{1}{n}\left[\eta^{+}-\left(\eta^{+}-\eta^{-}\right) \frac{i-1}{n-1}\right]\left(1 \leq \eta^{+} \leq 2, \eta^{-}=2-\eta^{+}\right)
$$

\section{Crossover operator}

According to the probability $p_{c}$, two individuals are selected randomly for crossover. Because the observation time calculated together with observation sequence is independent, the crossover point should only operate in the position of observation sequence. In order to make sure that the same natural number should not value in one sub chromosome. It is said that the sensor would not observe the same target twice. Therefore, some process need be done as follow: if the crossover point is between in two sub chromosome, operate crossover directly; If inside the chromosome, copy the content before the cross point to the next generation, and then scan the sub chromosome from beginning. If the number of target has appeared, transpose its related time. Otherwise, copy the number of target together with its related time.

But if the number of target is 0 , copy directly. The method of cross can adopt single point crossover, two point crossover, multi point crossover and uniform crossover.

\section{Mutation operator}

According to the smaller probability $p_{m}$, a single individual can be selected. In this individual, a random number from 1 to $2 \cdot m \cdot n$ as mutation position can be produced at uniform probability. At mutation, if it refers to observation time, directly mutate. While, if it refers to the number of target, make sure the value mutated of number should not overflow. On this condition, if this new mutated number of target has appeared at front, transpose it. Other wish, directly mutate.

\section{3) Clone operation}

The top $q$ best-individuals would be selected to clone. And the number of individual clone which fitness is $f_{i}(x)$, can be calculated as follow.

$$
N_{i}=\left\lfloor N * f_{i}(x) / \sum_{j=1}^{n} f_{j}(x)\right\rfloor
$$

In the formula, clone size is $N ;\lfloor\rfloor$ refers to round down.

\section{4) Adaptive strategy}

Crossover probability is $p_{c}$, mutation probability is $p_{m}$. These two genetic parameters directly affect convergence and convergent speed of the algorithm. Adaptive strategy can make $p_{c}, p_{m}$ automatically change with fitness, can 
provide relative to a solution to the best $p_{c}, p_{m}$, adjustment for genetic parameters ${ }^{[17]}$ :

$$
\begin{aligned}
& p_{c}= \begin{cases}p_{c \min }-\frac{\left(p_{c \max }-p_{\text {cmin }}\right)\left(f^{\prime}-f_{\text {avg }}\right)}{f_{\text {max }}-f_{\text {avg }}} & f^{\prime} \geq f_{\text {avg }} \\
p_{c \max } \text { else }\end{cases} \\
& p_{m}= \begin{cases}p_{m \min }-\frac{\left(p_{m \max }-p_{\text {min }}\right)\left(f_{\max }-f\right)}{f_{\max }-f_{\text {avg }}} & f \geq f_{\text {avg }} \\
p_{m \max } & \text { else }\end{cases}
\end{aligned}
$$

In the formula, maximum crossover probability is $p_{c \max }$; minimum crossover probability is $p_{c \min }$; maximum probability of mutation is $p_{m \max }$; minimum probability of mutation is $p_{m \min }$; the maximum fitness of individuals in population is $f_{\max }$; average fitness of the population is $f_{a x y}$; the larger fitness of the two individuals will cross is $f^{\prime}$; individual fitness will variation is $f$.

\section{B. Algorithm Flow}

In order to realize the multi-sensor resource scheduling dynamically, the target list and related senor list are given as follows[19]:

Target list a: OB1 shows the targets which need sensors to track.

Target list b: OB2 shows the targets which are in process of being tracked.

Target list c: OB3 shows the target which have been tracked.

Sensor list a: SR1 shows the available sensors (including those interrupted)

Sensor list b: SR2 shows the working sensors.

Sensor list c: SR3 shows the free sensors.

Step 1: If the tracking system starting with nothing found and $\mathrm{OB} 1=\mathrm{NULL}$, the scheduling continues. When new targets are found, OB1and SR1 updates.

Step 2: estimating the time the algorithm spends and finding the available sensors. If $\mathrm{OB} 1=0$, shelving the work until OB1 $=1$. Then update OB2. The sensors track the target in terms of the flight time of it. If $\mathrm{OB} 1>1$, planning the resource scheduling with the use of SCGA and the list of SR1 and OB2.

Step 3: Execution as shown in Figure 2 of SCGA cycle, get the scheduling scheme.

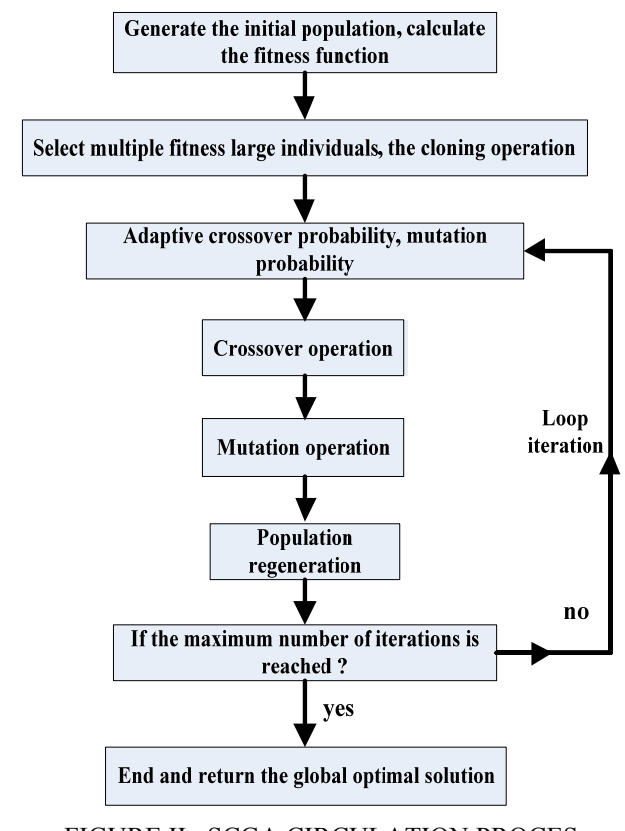

FIGURE II. SCGA CIRCULATION PROCES

Specific steps are as follows:

Step 3.1: Genetic algebra counter $g=0$. generate the initial population randomly, and evaluate the fitness function $f\left(x_{j}\right)$ of each individual in the population;

Step 3.2: select $\mathrm{n}$ individuals the fitness of which are the largest. These individuals composite of population $p_{n}$. Generate a temporary antibody set after cloning the population $p_{n}$;

Step 3.3: adaptively adjust the crossover probability $p_{c}$ and mutation probability $p_{m}$;

Step 3.4: select two individuals $x_{j}, x_{k}$ in the temporary antibody set randomly. Complete crossover operation with crossover probability $p_{c}$, and get new individuals $x_{j}^{\prime}, x_{k}^{\prime}$. Calculate the fitness $f\left(x_{j}^{\prime}\right), f\left(x_{k}^{\prime}\right)$;

If $\min \left\{1, \exp \left(\frac{-\left(f\left(x_{j}\right)-f\left(x_{j}^{\prime}\right)\right)}{T_{g}}\right)\right\}>$ random $[0,1]$

Then $x_{j}^{\prime}$ is received as a new individual If

$\min \left\{1, \exp \left(\frac{-\left(f\left(x_{k}\right)-f\left(x_{k}^{\prime}\right)\right)}{T_{g}}\right)\right\}>\operatorname{random}[0,1]$

Then $x_{k}^{\prime}$ is received as a new individual (this is metropolis rules). Random is a random number;

Step 3.5: According mutation probability $p_{m}$ doing the cloning operation with the individual ${ }^{X_{j}}$, and get new 
individuals ${ }^{x_{j}^{\prime}}$. Whether receiving the ${ }^{x_{j}^{\prime}}$ depends on the metropolis rules;

Step 3.6: $d$ individuals generated before alternative the worse individuals in the original population;

Step 3.7: If max-iterations is reached, the evolutionary process is over with the global optimal solution returned; or turn to step 3.3;

Step 4: Checkout the feasibility of scheduling according to all constraint conditions. The targets are assign to the correspond sensors. Update the list of OB2,OB3,SR2 and SR3. The targets which aren't assigned are put into OB1, waiting for the next scheduling.

Step 5 If tracking is not remove, repeat step 1 to step 4.

\section{Experimental Results}

In order to simplify the experiment, Simulation experiment can be done by the following scenario: 3 GEOS, 2 LEOS, and 2 ground surveillance radars. Their specific parameters are as follows[20].

Parameters of the GEOS: one scanning camera and one staring camera exist in each GEO, height of geostationary orbit is $36000 \mathrm{~km}$, positioning longitudes of three cameras are distributed uniformly, and the total visual field of camera is $15^{\circ}$.

Parameters of the LEOS: height is $1600 \mathrm{~km}$, one scanning camera and one capturing and tracking camera exist in each LEO. The parameters of scanning camera are designed as follows: size of visual field is $20^{\circ} \times 10^{\circ}$, detection speed is $1 \mathrm{~s}$, measuring error $\sigma_{L O S}$ is $50 \mu \mathrm{rad}$, capacity of targets is $100, \mathrm{SNR}$ is 5 . The parameters of capturing and tracking camera are designed as follows: size of visual field is $0.1^{\circ} \times 0.1^{\circ}$, detection speed is $30 \mathrm{~ms}$, measuring error $\sigma_{\text {LOS }}$ is $10 \mu \mathrm{rad}$, capacity of targets is 2 , SNR is 2 .

Parameters of ground surveillance radars: operating waveband is $\mathrm{P}$ band, detection range is $3000 \sim 5000 \mathrm{~km}$, observation of the airspace is $120^{\circ}$ in azimuth and $0^{\circ} \sim 85^{\circ}$ in pitch angle, capacity of targets is 30 .

Parameters of multi-functional array radars : modulation scheme is phased array, operating waveband is $\mathrm{X}$ band, detection range is $3000 \sim 5000 \mathrm{~km}$, observation of the airspace is $\pm 120^{\circ}$ in azimuth and $1^{\circ} \sim 90^{\circ}$ in pitch angle, capacity of targets is 100 in coarse tracking and 7 in accurate tracking, measure precision of range is $\sigma_{R}=30 \mathrm{~m}$ in coarse tracking and $5 \mathrm{~m}$ in accurate tracking, that of angle $\sigma_{\theta}$ is $0.05 \sim 0.1^{\circ}$, that of speed is $\sigma_{V} \leq 0.5 \mathrm{~m} / \mathrm{s}$, and that of range resolution $\Delta R$ is $300 \sim 350 \mathrm{~m}$ in narrow-band and $100 \mathrm{~m}$ in wide-band. The power is $600 \mathrm{KW}$ and interception probability is not less than $90 \%$.

Many simulation experiments have been done. There are $\mathrm{n}$ AHV in each experiment. The AHV can be observed by GEOS's scanning camera after flying for a period. And the noises variance is $5 \mu \mathrm{rad}$. After entering into the free flight phase, the targets can be detected and tracked by the LEOS. And the LEOS guides the ground-based radar to track the target. Through setting boost time of the AHV, time-offlight of target that crosses the monitoring field of each sensor node can be got by the software of STK. As a result the space problem can be converted into the time problem.

This paper set the simulation parameters algorithm as follows: Population size pop $=100$, maximum iterations number $\quad \mathrm{max}=20$, clone size $\mathrm{N}=100$, $\operatorname{pcmax}=0.9$, pmmax $=0.4, \operatorname{pcmin}=0.1, \mathrm{pmmin}=0.01, \mathrm{~d}=10$. By AHP to get evaluation index weight of objective function value, set as $w_{1}=0.55,{ }^{w_{2}}=0.25,{ }^{w_{3}}=0.15, w_{4}=0.05$.

Simulation uses these three kinds of algorithms to test independently 100 times. And average value of results about each algorithm is given to compare. Details are as shown in the table 1 and table 2 :

TABLE I. RESULTS OF THE THREE ALGORITHM

\begin{tabular}{|c|c|c|c|c|c|c|}
\hline \multirow{2}{*}{$\begin{array}{c}\text { Numbe } \\
\text { r of } \\
\text { targets }\end{array}$} & $\begin{array}{c}\text { Number } \\
\text { of } \\
\text { iteration } \\
\boldsymbol{s}\end{array}$ & fitness & $\begin{array}{c}\text { Sum } \\
\text { ber } \\
\text { of } \\
\text { iterat } \\
\text { ions }\end{array}$ & fitness & $\begin{array}{c}\text { Num } \\
\text { ber } \\
\text { of } \\
\text { iterat } \\
\text { ions }\end{array}$ & fitness \\
\hline 2 & 45 & 0.9153 & 53 & 0.8999 & 49 & 0.9065 \\
\hline 4 & 86 & 0.9000 & 95 & 0.8980 & 92 & 0.8975 \\
\hline 6 & 98 & 0.8991 & 121 & 0.8763 & 110 & 0.8678 \\
\hline 8 & 105 & 0.8989 & 136 & 0.8678 & 126 & 0.8662 \\
\hline 10 & 109 & 0.8992 & 152 & 0.8663 & 131 & 0.8620 \\
\hline
\end{tabular}

TABLE II. RESULTS OF THE THREE ALGORITHM

\begin{tabular}{|c|c|c|c|}
\hline \multirow{2}{*}{$\begin{array}{c}\text { Number } \\
\text { of } \\
\text { targets }\end{array}$} & SCGA & CLONALG & GA \\
\hline & $\begin{array}{c}\text { Average running } \\
\text { time }\end{array}$ & $\begin{array}{c}\text { Average running } \\
\text { time }\end{array}$ & $\begin{array}{c}\text { Average running } \\
\text { time }\end{array}$ \\
\hline 2 & 4.8163 & 5.8573 & 6.1367 \\
\hline 4 & 5.2362 & 6.4523 & 7.3237 \\
\hline 6 & 6.7464 & 7.6877 & 8.7824 \\
\hline 8 & 8.3532 & 9.1525 & 10.4625 \\
\hline 10 & 10.2312 & 10.8793 & 12.3527 \\
\hline
\end{tabular}

It can be seen that, compared with CLONALG and GA, when the number of targets is the same, iterations of SCGA is smaller than those. And the objective function value is relatively large. As the targets quantity increased, The increase of SCGA iterations number is relatively small, the decrease of the fitness function value is relatively small. For instance: When the targets number is 4 , iteration number of SCGA is less than CLONALG 8 times, less than GA 4 times; Fitness function value is more than GLONALG $1.7 \%$, more than GA $0.8 \%$. When the targets number is 10 , iteration number of SCGA is less than CLONALG 17 times, less than GA 40 times; Fitness function value is more than GLONALG 3.8\%, more than GA $4.3 \%$. Specify the target numbers increase, SCGA effect is better. In order to visually compare change regulation of fitness with iterations, When the target number are 4 and 10, Based on MATLAB 
simulation obtained generations of fitness variation curve in Figure 3 and Figure 4.

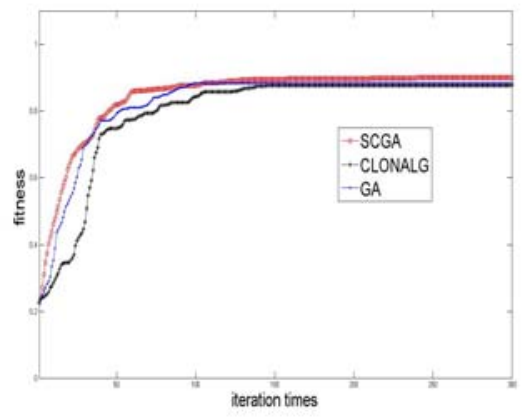

FIGURE III. THE MAXIMUM FITNESS CURVES IN 4 NUMBERS

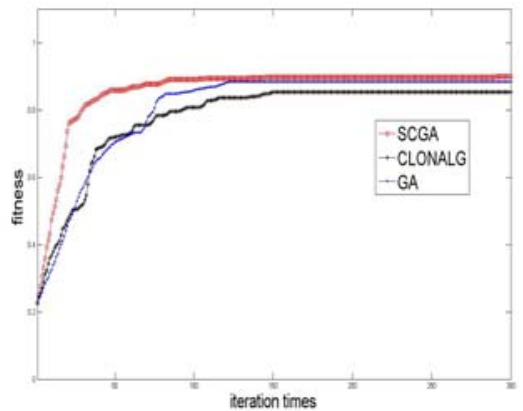

FIGURE IV. THE MAXIMUM FITNESS CURVES IN 10 NUMBERS

The Figure 3 and Figure 4 show, Compared with GA and CLONALG, SCGA search ability is the most outstanding, possesses good convergence speed and accuracy. When target number is increased, the simulation results show that search ability of SCGA is particularly prominent. Better able to meet a certain number of sensors for collaborative tracking of AHV .

\section{ACKNOWLEDGMENT}

This work was supported by National Natural Science Foundation of China(No. 61272011 and 61102109).

At the point of finishing this paper, I'd like to express my sincere thanks to all those who have lent me hands in the course of my writing this paper. First of all, I'd like to take this opportunity to show my sincere gratitude to my supervisor, who has given me so much useful advice on my writing, and has tried her best to improve my paper. Secondly, I'd like to express my gratitude to my classmates who offered me references and information on time. Last but not the least, I'd like to thank those leaders, teachers and working staff especially those in the School of Foreign Languages. Without their help, it would be much harder for me to finish my study and this paper.

\section{REFERENCES}

[1] Wang W Q. "Near-space vehicle-borne SAR with reflector antenna for high-resolution and wide-swath remote sensing".
Geoscience and Remote Sensing, IEEE Transactions on 50.2 (2012): 338-348

[2] Markin, Evgeny. "Scatter pattern calculations and determination performance limitations of existing air defense systems for intercepting hypersonic vehicles". Radar Conference (RADAR), 2012 IEEE. IEEE, 2012.

[3] Naseh, H, M. Mirshams. "A Bayesian networks approach to reliability analysis of a space vehicle separation sub-system". Recent Advances in Space Technologies (RAST), 2013 6th International Conference on. IEEE, 2013.

[4] Yang, Qingyun, and Mou Chen. "Composite nonlinear control for near space vehicles with input saturation based on disturbance observer." Control Conference (CCC), 2013 32nd Chinese. IEEE, 2013

[5] Moran W, Howard S D, Suvorova S. Sensor management via Riemannian geometry[C]//Proc. of the Communication, Control, and Computing, 2012: 358-362.

[6] De A, Koditschek D E. "Toward dynamical sensor management for reactive wall-following". Proc. of the IEEE International Conference on Robotics and Automation, 2013:2400-2406.

[7] Newstadt G, Hero A O. Sensor management and provisioning for multiple target radar tracking systems $[\mathrm{C}] /$ Proc. of the IEEE International Conference on Acoustics, Speech and Signal Processing, 2012: 5273-5276.

[8] Evers, Lanah, et al. "Online stochastic UAV mission planning with time windows and time-sensitive targets". European Journal of Operational Research 238.1 (2014): 348-362.

[9] Höckerdal, Erik, Erik Frisk, and Lars Eriksson. "EKF-based adaptation of look-up tables with an air mass-flow sensor application." Control Engineering Practice 19.5 (2011): 442-453.

[10] Wu L F, Wang G, Liu C Y. "Task programming of antiballistic missile sensors based on MAS". Modern Defence Technology, 2012, 40(3): 88-93.

[11] Sun, Haibin, Shihua Li, and Changyin Sun. "Finite time integral sliding mode control of hypersonic vehicles". Nonlinear Dynamics 73.1-2 (2013): 229-244.

[12] Osadciw L, Veeramache-neni K. A "controllable sensor management algorithm capable of learning". Proc. of the SPIE, 2005.

[13] Morgan U, Jonas N. A sensor Management Framework for Autonomous UAV Surveillance[C]/Proc. of the SPIE, 2005.

[14] Ferber J. Multi-agent systems: an introduction to distributed artificial intelligence[M]. Reading: Addison-Wesley, 1999.

[15] Tom S. A neural network approach to dynamic task assignment of multirobots[J]. IEEE Trans. on Neural Networks, 2006, 75(5): 1278-1287.

[16] Zuo L, Wang M L, Yang Y. "Complex terrain classification algorithm based on multi-sensors fusion". Proc. of the Control Conference(CCC), 2013: 5722-5727.

[17] Bentley E S, Kondi L P, Matyjas J D. Effective resource management in visual sensor networks with MPSK[J]. Signal Processing Letters, 2013:739-742.

[18] Pourvaziri, Hani, and B. Naderi. "A hybrid multi-population genetic algorithm for the dynamic facility layout problem." Applied Soft Computing (2014).

[19] Chen, Fafa, Baoping Tang, and Renxiang Chen. "A novel fault diagnosis model for gearbox based on wavelet support vector machine with immune genetic algorithm." Measurement 46.1 (2013): 220-232.

[20] Guo H B, Wang Y L, Zeng H. "Sensor scheduling for missile early-warning satellite based on genetic and simulated annealing algorithm". Electronics Optics \& Control. 2006, 13(4):71-74. 